\title{
UMA AVALIAÇÃO DE DESCRITORES DE TEXTURA BASEADOS EM CÓDIGOS BINÁRIOS LOCAIS PARA CLASSIFICAÇÃO DE IMAGENS DE SENSORIAMENTO REMOTO
}

\author{
An evaluation of texture descriptors based on local binary patterns for \\ classifications of remote sensing images \\ MARCELO MUSCI ${ }^{1}$ \\ RAUL QUEIROZ FEITOSA ${ }^{1}$ \\ MARIA LUIZA FERNANDES VELLOSO ${ }^{2}$ \\ TESSIO NOVACK ${ }^{3}$ \\ GILSON ALEXANDRE OSTWALD PEDRO DA COSTA ${ }^{1}$ \\ ${ }^{1}$ Pontifícia Universidade Católica do Rio de Janeiro - PUC-Rio \\ ${ }^{2}$ Universidade Estadual do Rio de Janeiro - UERJ \\ ${ }^{3}$ Instituto Nacional de Pesquisas Espaciais - INPE \\ ${ }^{1}$ \{musci, raul, gilson\}@ele.puc-rio.br \\ 2mlfv@centroin.com.br \\ 3tessio@dsr.inpe.br
}

\begin{abstract}
RESUMO
O presente trabalho avalia descritores texturais multiescalares invariantes à rotação baseados em Padrões Binários Locais e em Quantização de Fase Local (LPQ) para a classificação de uso e cobertura do solo em uma imagem IKONOS-2 e uma imagem Quickbird-2. Os experimentos mostraram que ambas as representações de textura propiciaram elevada acurácia quando combinadas com a informação de variância. Além disso, propõe-se no trabalho um novo descritor formado pela concatenação do histograma de variância e o histograma dos códigos gerados a partir dos Padrões Binários Locais ou da Quantização de Fase Local. Com o novo descritor, não obstante serem comparativamente mais compacto, registraram-se os mesmos índices de desempenho obtidos a partir de histogramas bidimensionais que representam a distribuição conjunta de ambas as variáveis. $O$ último experimento realizado indicou para os descritores baseados nos Padrões Binários Locais ou na Quantização de Fase
\end{abstract}


Local um índice Kappa superior em 0,1 ao que alcançou quando se utilizaram atributos de textura derivados da matriz de co-ocorrência.

Palavras-chave: Textura; LBP; LPQ; GLCM; Sensoriamento Remoto.

ABSTRACT.
In this paper rotation invariant, single and multiscale Local Binary Patterns and Local Phase Quantization texture based descriptors were evaluated experimentally in the context of land-use and land-cover object-based classification. The texture descriptors were employed in the classification of an IKONOS-2 and a Quickbird-2 image. Experiments showed that both texture characterization approaches perform well, when combined with the grayscale variance. We further investigate a novel descriptor resulting from the concatenation of the grayscale variance histogram and the histogram of codes generated either by Local Binary Patterns or by Local Phase Quantization. These experiments have demonstrated that the proposed descriptor, though more compact, performs as well as a bidimensional histogram representing the joint distribution of both quantities. A final experiment corroborated that the use of descriptors based on Local Binary Patterns or Local Phase Quantization in the remotely sensed images classification delivered produces a 0.1 improvement in the Kappa index in comparison to classifications based on texture features derived from the Gray Level Co-occurrence Matrix (GLCM).

Keywords: Texture; LBP; LPQ; GLCM; Remote Sensing.

\section{INTRODUÇÃO}

A partir do final da década de 90 tornaram-se disponíveis comercialmente imagens de satélite de muito alta resolução espacial, frequentemente denotadas pela sigla VHR (do inglês very high resolution), com pixels de dimensão entre $0,5 \mathrm{~m}$ e 1 m (LU; WENG, 2007). Técnicas tradicionais de classificação pixel-a-pixel demonstraram-se ineficientes para a interpretação de imagens com tal resolução, sobretudo devido à grande variabilidade dos atributos espectrais dentro de uma mesma classe. Essa limitação fez crescer a partir de então o interesse pela técnica de análise de imagens baseada em objeto, conhecida pelos acrônimos OBIA (do inglês Object Based Image Analysis) ou GEOBIA (do inglês Geographic Object Based Image Analysis) (JYOTHI; BABU; KRISHNA, 2008; HAY; CASTELLAN, 2008). As virtudes da OBIA foram comprovadas ao longo da última década em inúmeras aplicações, como indica, por exemplo, o extenso levantamento bibliografico conduzido por Blaschke (2010). Tal sucesso decorre sobretudo de se considerarem nesta abordagem atributos estruturais, de forma e textura, além de atributos espectrais, e, ao mesmo tempo da capacidade de emular a habilidade humana de delinear e identificar objetos de interesse nas imagens.

Essencialmente, OBIA consiste de dois passos principais. Primeiramente a segmentação, que subdivide a imagem em regiões homogêneas, e, por fim, a classificação de cada um dos segmentos gerados no primeiro passo. Tanto a 
segmentação quanto a classificação são guiadas por critérios baseados em atributos das regiões da imagem. Entre estes, a informação de textura tem papel relevante, como se demonstrou em várias aplicações, tais como identificação de culturas agrícolas (PEÑA-BARRAGÁN et al., 2011), classificação de uso e cobertura do solo em áreas urbanas (BOSCHI; GALO, 2007) (ZHANG; ZHU, 2011), mapeamento florestal (KIM; MADDEN; WARNER, 2009), extração automática de redes viárias (WANG; HU; ZHANG, 2005), entre outras. Nestas aplicações, como de resto na maioria das aplicações de sensoriamento remoto (SR), atributos calculados a partir das matrizes de co-ocorrência (Gray Level Co-ocorrence Matrix - GLCM), (HARALICK, 1973) preponderam sobre outros descritores de textura propostos na literatura (PETROU; SEVILLA, 2006).

Recentemente um novo método para representação de texturas baseado em códigos binários locais, denominada Local Binary Patterns (LBP), foi proposto inicialmente em (OJALA; PIETIKÄINEN; HARWOOD; 1996) e mais tarde generalizado em (OJALA; PIETIKÄINEN; MÄENPÄÄ, 2002). O método alcançou notável sucesso nas aplicações de reconhecimento facial (AHONEN; HADID; PIETIKÄINEN, 2006). Ojala, Pietikäinen e Mäenpää (2002) apresentaram um estudo que avaliou LBP na caracterização de texturas utilizando o albúm de Brodatz (1966) como base de dados, com resultados igualmente encorajadores.

Entretanto, na área de SR são poucos os trabalhos que utilizam o LBP, e entre estes a maioria é voltada à segmentação, por exemplo, em (WANG, A.; WANG, S.; LUCIEER, 2010; ORKHONSELENGE, 2004; LUCIEER; STEIN; FISHER, 2005). Ainda mais raras são as publicações sobre classificação de imagens de SR em que se usa o LBP para caracterizar texturas. Uma exceção é o trabalho de Song, Yang e Li (2010) que testa o LBP em um mosaico de imagens de SR. Em que pesem os resultados positivos alcançados neste trabalho, a avaliação se ressente de ter sido realizada apenas sobre imagens sintéticas.

$\mathrm{Na}$ trilha do sucesso do LBP, um novo método de representação de textura também baseado em códigos binários locais foi proposto em (AHONEN et al., 2008). O método denominado Local Phase Quantization (LPQ) demonstrou-se superior ao LBP nas aplicações de reconhecimento facial. Ojansivu e Heikkilä (2008); Heikkilä e Ojansivu (2009); Heikkilä, Ojansivu e Rahtu (2010) utilizaram o LPQ na análise de textura de imagens sintéticas, Chan et al. (2009) fez uso de uma abordagem do LPQ em diferentes escalas em reconhecimento facial. Os autores do presente trabalho desconhecem, no entanto, qualquer estudo publicado em que o LPQ tenha sido avaliado na classificação de imagens de SR.

O objetivo do trabalho descrito neste artigo foi avaliar o desempenho do LBP e do LPQ como descritores de textura em aplicações reais de análise de imagens de $\mathrm{SR}$, seguindo a abordagem baseada em objeto. Avaliam-se neste estudo diferentes configurações multiescalares do LBP e LPQ invariantes à rotação para imagens VHR, usando como referência os descritores baseados em GLCM. A avaliação é conduzida em uma seqüência de experimentos utilizando dois conjuntos de dados. $\mathrm{O}$ primeiro conjunto de dados é composto por uma imagem Quickbird-2 e o respectivo 
experimento explora a classificação de tipos de assentamentos urbanos, como favelas e áreas urbanas de alta/baixa renda. O segundo conjunto de dados é composto por uma imagem IKONOS-2 e o experimento consiste na identificação de classes de cobertura do solo.

O presente trabalho aprofunda a análise preliminar publicada em (MUSCI et al., 2011), inclui o LPQ na avaliação e utiliza um classificador mais poderoso baseado na técnica de Support Vector Machine (SVM) - para comparar os descritores baseados em códigos locais com os descritores derivados da GLCM. Além disso, propõe-se neste trabalho um novo descritor de textura que combina o LBP ou o LPQ com a informação da variância local.

As seções que se seguem estão organizadas da seguinte maneira. Uma breve descrição das principais variantes do LBP e do LPQ, bem como da estratégia de classificação subjacente, é apresentada na Seção 2. A Seção 3 descreve os experimentos realizados, apresenta e discute os resultados obtidos. Um resumo das principais conclusões e direções futuras são apresentados na Seção 4.

\section{CLASSIFICAÇÃO DE TEXTURA}

Esta seção descreve os atributos texturais avaliados neste trabalho e a técnica mais comumente utilizada para medir similaridade entre texturas descritas por códigos binários locais.

\subsection{Codificação de Textura via LBP}

O LBP associado a um pixel na posição $\mathbf{w}=(x, y)$ é calculado a partir de um conjunto de $P$ amostras igualmente espaçadas e distribuídas sobre uma circunferência de raio $R$ com centro sobre o pixel, como ilustrado na Figura 1. A partir das intensidades $g_{p}$ de cada uma destas amostras, onde $0 \leq p<P$, e da intensidade $g_{c}$ do próprio pixel na posição $\mathbf{w}$, obtém-se uma seqüência de $P$ valores binários $T_{P}=\left\{S\left(g_{0}-g_{c}\right), S\left(g_{1}-g_{c}\right), \ldots, S\left(g_{P-1}-g_{c}\right)\right\}$, onde $S$ é a função sinal, ou seja, é nula quando o argumento é negativo e igual a 1 caso contrário.

Um procedimento simples mapeia a seqüência de bits $T_{P}$ em um valor inteiro. Determinou-se empiricamente que entre as possíveis seqüencias $T_{P}$, aquelas em que ocorrem no máximo duas transições de 0 para 1 e de 1 para 0 são as mais importantes para a caracterização de texturas (OJALA; PIETIKÄINEN; MÄENPÄÄ, 2002). Define-se a partir daí um código $L B P_{P, R}$ invariante à rotação, como sendo o número de 1 's da seqüência. Cria-se adicionalmente um código especial para as seqüências em que ocorrem mais do que duas transições de 1 para 0 ou de 0 para 1, (Ojala et al., 2002). Formalmente:

$$
L B P_{P, R}(\mathbf{w})=\left\{\begin{array}{c}
\sum_{p=0}^{P-1} S\left(g_{p}-g_{c}\right) \text { se número de transições } \leq 2 \\
P+1 \text { caso contrário }
\end{array}\right.
$$


O $L B P_{P, R}$, pode assumir um entre $P+2$ valores distintos que representa o arranjo espacial dos tons de cinza (a textura, portanto) na vizinhança em torno de cada pixel. Claramente, o $L B P_{P, R}$ é invariante contra transformações monotônicas da escala de cinza. Alterando-se os valores de $P$ e $R$ é possível representar a textura em outras escalas.

Figura 1 - Representação do LBP para diferentes valores de $P$ e $R$.

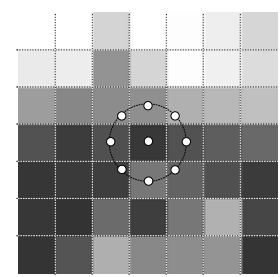

$P=8, R=1$

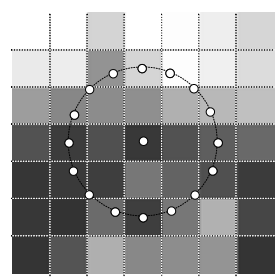

$P=16, R=2$

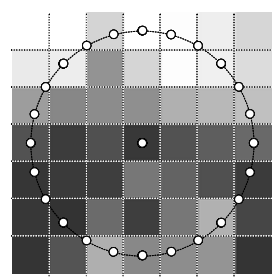

$P=24, R=3$

\subsection{Codificação de Textura via LPQ}

O LPQ é um descritor de texturas concebido para superar o LBP em aplicações onde as imagens estão borradas ou afetadas por iluminação não uniforme. De forma similar ao LBP, para cada pixel na posição $\mathbf{w}=(x, y)$ um código é computado para representar a textura de pixels numa vizinhança $\mathrm{N}_{\mathrm{w}}$, centrada em $\mathbf{w}$, de tamanho $M \times M$ pixels, como a mostrada na Figura $2 \mathrm{~A}$.

A quantização de fase leva em conta apenas o sinal das componentes real e imaginária da transformada de Fourier $\mathbf{F}_{\mathbf{w}}(\mathbf{u}), \mathbf{u}=(u, v)$, em pelo menos quatro valores de freqüências próximos à origem, especificamente $(0, a),(a, a),(a, 0)$ e $(a$,$a$ ), conforme indicam os círculos brancos na Figura $2 \mathrm{~B}$, onde $a=1 / M$. São gerados assim 8 bits, que assumem o valor 0 ou 1, dependendo de cada valor ser negativo ou não-negativo. Estes bits são concatenados em uma ordem arbitrária porém fixa, formando um valor inteiro de 8 bits que representa a textura em $\mathrm{N}_{\mathrm{w}}$. Este procedimento é executado para todos os pixels na imagem, formando a correspondente imagem $L P Q_{M}$, onde $M \times M$ representa o tamanho da vizinhança em pixels.

A lógica por trás do método pode ser entendida em linhas gerais levando-se em conta algumas propriedades da Transformada de Fourier. Considerando que $\mathbf{F}_{\mathbf{w}}(\mathbf{u})$ contém toda a informação presente em $\mathrm{N}_{\mathbf{w}}$, a idéia consiste em extrair de $\mathbf{F}_{\mathbf{w}}(\mathbf{u})$ um código que seja compacto e ao mesmo tempo capture o máximo da informação da textura em $\mathrm{N}_{\mathrm{w}}$.

É fato bem conhecido que a informação contida em $\mathbf{F}_{\mathbf{w}}(\mathbf{u})$ se concentra nas freqüências mais baixas. Sabe-se ainda que o valor da Transformada de Fourier na origem corresponde ao brilho médio da vizinhança, o que, para efeitos de textura, é irrelevante. Assim, as componentes indicadas na Figura 2B e suas simétricas são as 8 componentes de $\mathbf{F}_{\mathbf{w}}(\mathbf{u})$ onde se concentra a maior parte da informação da textura 
na vizinhança. Os 8 valores complexos assim obtidos podem ser reduzidos a 4 sem perda de informação, tendo em vista que os valores da Transformada de Fourier em posições simétricas são conjugados, ou seja, $\mathbf{F}_{\mathbf{w}}(-\mathbf{u})=\mathbf{F}_{\mathbf{w}}{ }^{*}(\mathbf{u})$, e carregam, portanto, informação redundante. Justifica-se, assim, a seleção das 4 amostras indicadas na figura $2 \mathrm{~B}$. Este conjunto de 4 números complexos pode ser ainda mais compactado com pequena perda de informação, considerando que a fase (e não a magnitude) captura a maior parte da informação da Transformada de Fourier. Destarte, os 4 números complexos são substituídos pelos correspondentes valores de fase, transformando-se em 4 números reais. O último passo é a quantização de fase, em que o valor real que representa a fase é substituído por um código de dois dígitos binários indicativo do respectivo quadrante do plano complexo. O código se infere dos sinais das componentes real e imaginária de $\mathbf{F}_{\mathbf{w}}(\mathbf{u})$. Desta maneira, cada par de bits do código LPQ meramente identifica o quadrante em que se encontram as componentes da Transformada de Fourier indicadas na Figura 2B. Os 8 bits assim obtidos compõem o código que representa a textura na vizinhança.

$\mathrm{O}$ método inclui ainda um procedimento simples que decorrelaciona os coeficientes de Fourier antes da etapa de quantização. Este visa a maximizar a informação preservada no código da textura. Uma descrição detalhada do método é apresentada em (OJANSIVU; HEIKKILÄ, 2008).

Figura 2 - Imagem de entrada de pixels vizinhos $\mathrm{N}_{\mathrm{w}}(\mathrm{A})$ e o módulo da Transformada de Fourier $\left|\mathbf{F}_{\mathbf{w}}(\mathbf{u})\right|$ da imagem de entrada $\mathrm{N}_{\mathbf{w}}(\mathrm{B})$.
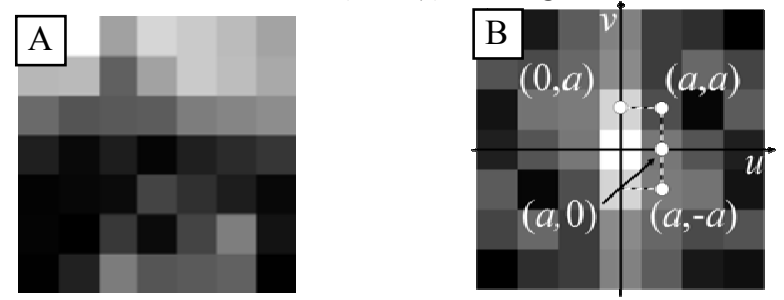

$\mathrm{Na}$ análise de imagens de SR é importante que os descritores apresentem invariância quanto à rotação. Uma versão do LPQ invariante à rotação é descrita por Ojansivu, Rahtu e Heikkilä (2008). Esta proposta prevê um passo preliminar que calcula o ângulo correspondente à chamada orientação característica da vizinhança. Aplica-se à vizinhança em torno de cada pixel uma rotação no valor do ângulo calculado no primeiro passo. O procedimento já descrito que gera os códigos LPQ é aplicado em seguida à vizinhança rotacionada, produzindo, desta forma, o código binário invariante à rotação que representa a textura naquela vizinhança.

É possível descrever a textura em múltiplas escalas calculando-se a imagem $L P Q_{M}$ para diferentes valores de $M$. 


\subsection{Codificação de Textura via VAR}

Os descritores LBP são, por definição, invariantes quanto a transformações monotônicas de níveis de cinza e não capturam, portanto, o contraste local. Ojala, Pietikäinen e Mäenpää (2002) propõem um descritor do contraste local, denotado pelo símbolo $V A R_{P, R}$, também invariante a rotação, como definido na equação (2) a seguir:

$$
\operatorname{VAR}_{P, R}(\mathbf{w})=\frac{1}{P} \sum_{p=0}^{P-1}\left(g_{p}-\mu\right)
$$

onde $\mu=\frac{1}{P} \sum_{p=0}^{P-1} g_{p} . V A R_{P, R}$ é uma aproximação da variância local, com a vantagem de que e o esforço computacional para calculá-lo é comparativamente menor, especialmente quando calculado simultânemente com o $L B P_{P, R}$.

Assim, uma vez definidos os valores de $P$ e $R$, pode-se calcular de modo eficiente para cada pixel da imagem dois códigos, um representando a textura $\left(L B P_{P, R}\right)$ e o outro o contraste $\left(V A R_{P, R}\right)$ na vizinhança em torno do pixel.

\subsection{Similaridade entre Texturas}

A textura de um segmento da imagem pode ser caracterizada a partir do histograma normalizado dos códigos binários locais calculados para cada um dos pixels pertencentes ao segmento. Este histograma é considerado como uma assinatura da textura dominante no interior do segmento. Analogamente, cada classe do sistema é descrita por um histograma modelo calculado a partir de um conjunto representativo de amostras de segmentos da classe. A comparação entre a textura de um segmento e a textura característica de uma classe se faz por meio de uma métrica que expressa numericamente a similaridade entre histograma do segmento e o histograma modelo.

$\mathrm{Na}$ maioria dos trabalhos relacionados mede-se a similaridade entre dois histogramas utilizando a estatística G (SOKAL; ROHLF, 1987). A distribuição conjunta $L B P_{P, R} / V A R_{P, R}$ representada em histogramas bidimensionais foi utilizada para a segmentação e classificação de imagens de sensoriamento remoto, como se encontra, por exemplo, em (RUBIO et al., 2001; ORKHONSELENGE, 2004; LUCIEER; STEIN, 2005; GUO; ATLURI; ADAM, 2005; HU; TAO; PRENZEL, 2005; SONG; YANG; LI, 2010; WANG, S.; WANG, A., 2008). Na maioria destes trabalhos mede-se a similaridade entre dois histogramas utilizando a estatística $\mathrm{G}$, definida na equação (3)

$$
\begin{gathered}
\mathrm{G}\left(f_{1}, f_{2}\right)= \\
2\left[\begin{array}{c}
\sum_{i=1}^{B} f_{1}(i) \log f_{1}(i)+\sum_{i=1}^{B} f_{2}(i) \log f_{2}(i)-\left(\sum_{i=1}^{B} f_{1}(i)\right) \log \left(\sum_{i=1}^{B} f_{1}(i)\right)- \\
\left(\sum_{i=1}^{B} f_{2}(i)\right) \log \left(\sum_{i=1}^{B} f_{2}(i)\right)-\sum_{i=1}^{B}\left(\left(f_{1}(i)\right)+f_{2}(i)\right) \log \left(f_{1}(i)+f_{2}(i)\right)+ \\
\left(\sum_{i=1}^{B} f_{1}(i)+\sum_{i=1}^{B} f_{2}(i)\right) \log \left(\sum_{i=1}^{B} f_{1}(i)+\sum_{i=1}^{B} f_{2}(i)\right)
\end{array}\right]
\end{gathered}
$$


onde $f_{1}$ e $f_{2}$ são os histogramas da amostra e do modelo respectivamente e $B$ é o número de bins dos histogramas $f_{1}$ e $f_{2}$. É fácil verificar que quanto mais similares forem os histogramas, menor será o valor de $G$.

Quando se opera com múltiplas escalas, a similaridade entre texturas é dada pela soma das similaridades dos histogramas em escalas correspondentes, ou seja.

$$
\mathrm{G}_{\text {multi }}=\sum_{s_{i}} \mathrm{G}\left({ }^{s_{i}} f_{1},{ }^{s_{i}} f_{2},\right)
$$

onde ${ }^{s_{i}} f_{1}$ e ${ }^{s_{i}} f_{2}$ correspondem aos histogramas da amostra e do modelo, obtidos com os parâmetros de escala $s_{i}$, definidos no LBP e no VAR pelos parâmetros $P$ e $R$ e no LPQ pelo parâmetro $M$.

No último da série de experimentos relatados a partir da próxima seção utilizase uma SVM (CORTES; VAPNIK, 1995), que tem sido utilizado de modo crescente em aplicações de SR (HUANG; DAVIS; TOWNSHED, 2002; BAZI; MELGANE, 2006; PAL; FOODY, 2010). O propósito neste experimento foi comparar códigos binários locais com atributos derivados da GLCM, para o que a SVM se mostra mais adequada do que a estatística $\mathrm{G}$.

\section{AVALIAÇÃO EXPERIMENTAL}

Esta seção descreve o procedimento e resultados de uma seqüência de experimentos que visaram avaliar os descritores de textura considerados neste trabalho.

\section{1 Áreas de Estudo}

\subsection{1 Área 1 - Classificação de Uso}

A primeira área alvo localiza-se na cidade de São Paulo. A imagem de entrada foi capturada em 31 de março de 2002 pelo sensor Quickbird-2 com resolução de $0,6 \mathrm{~m}$ na banda pancromática e dimensão de $4000 \times 4000$ pixels (coordenadas Norte/Leste 322464/7389053 Sul/Oeste 324872/7386671, projeção UTM-SAD69).

Trata-se neste caso de classificação de uso do solo. Os objetos a serem classificados são quadras cujos limites foram extraídos de dados cadastrais da Secretaria Municipal de Planejamento Urbano de São Paulo (PMSP, 2009). A Figura 3A mostra uma composição RBG da imagem utilizada.

As classes do problema são Urbana alta renda, Urbana Vertical, Urbana baixa renda, Favela, Terrenos desocupados. Devido à grande semelhança que se observa na imagem optou-se neste experimento por combinar as classes Urbana alta renda e Urbana Vertical numa classe única. A Figura 3B mostra a classificação também fornecida pela prefeitura e utilizada como verdade de campo nos experimentos. A Tabela 1 apresenta o número de quadras pertencentes a cada classe do problema. 
Figura 3 - Imagem Quickbird-2 utilizada no experimento.

Classificação de uso (A); mapa de uso do solo (B).
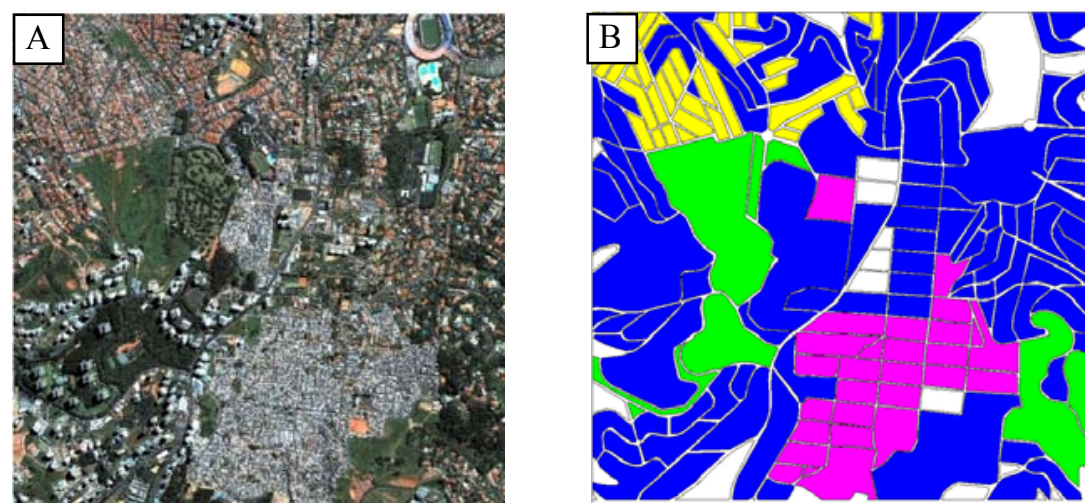

Urbana alta renda + Urbana Vertical $\square$ Favela

Urbana baixa renda $\square$ Terrenos desocupados

Tabela 1 - Distribuição de segmentos entre as classes de uso do solo

\begin{tabular}{lc}
\hline \multicolumn{1}{c}{ Classe } & Número de Segmentos \\
\hline Favela & 25 \\
Urbana alta/vertical & 101 \\
Terrenos desocupados & 8 \\
Urbana de baixa renda & 42 \\
\hline
\end{tabular}

\subsection{2 Área 2 - Classificação de Cobertura}

Trata-se de uma área localizada no município do Rio de Janeiro recoberta por uma imagem IKONOS-2 pancromática com resolução de $1 \mathrm{~m}$ capturada em 30 de maio de 2010 (coordenadas Norte/Leste 7471911/669457 e Sul/Oeste 7453904/688804, UTM-SAD 69, WGS 1984), com dimensão $2800 \times 2000$ pixels.

A Figura 4A mostra uma composição RGB da imagem fusionada com as bandas multiespectrais. Toda a área mostrada na Figura 4A foi segmentada utilizando um programa comercial, e os segmentos resultantes foram classificados visualmente, produzindo o mapa mostrado na Figura 4B que apresenta também as classes do problema, isto é, Campo, Floresta e Urbana. Trata-se, portanto de um problema de classificação de cobertura do solo. A Tabela 2 traz informações adicionais sobre o mapa que foi usado como referência. 
Figura 4 -Área da cidade do Rio de Janeiro IKONOS-2 (A); mapa uso do solo (B)
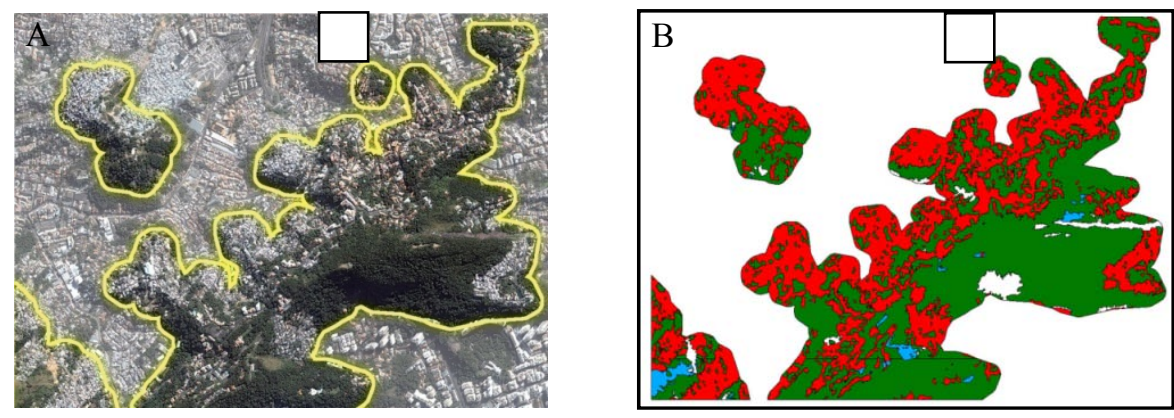

$$
\text { Campo } \quad \text { Flore } \square \quad \text { Urb } \square
$$

Tabela 2 - Distribuição de segmentos entre as classes de cobertura do solo

\begin{tabular}{cc}
\hline Classe & Número de Segmentos \\
\hline Campo & 5 \\
Floresta & 57 \\
Urbana & 26 \\
\hline
\end{tabular}

\subsection{Experimentos}

Como o objetivo primário do estudo não foi maximizar a acurácia da classificação, mas comparar o desempenho associado a cada descritor, optou-se em todos os experimentos por classificar as imagens de entrada baseando-se exclusivamente em atributos de textura. Muito provavelmente a inclusão de atributos espectrais ou de forma levaria a índices de desempenho mais elevados, por outro lado, implicaria num aumento da complexidade da etapa de classificação. Por conta disso, os objetos foram descritos em nossos experimentos tão somente em termos de atributos texturais.

Como se demonstra mais adiante no texto, o valor dos parâmetros $P$ e $R$ no LBP e do parâmetro $M$ no $L P Q$ têm impacto sobre a acurácia da classificação. Os valores ótimos destes parâmetros estão relacionados às dimensões das estruturas periódicas que, em última análise, caracterizam as texturas presentes na imagem. A escala de interesse é, portanto, crucial. Assim, os parâmetros de cada abordagem devem ser ajustados para cada aplicação particular, o que na maioria dos casos requererá alguma experimentação.

Em nossos experimentos foram testadas várias combinações de valores dos parâmetros $P$ e $R$ para os descritores $L B P_{P, R}$ e $V A R_{P, R}$ e diferentes tamanhos da janela de $M \times M$ pixels onde se calcula o código binário $L P Q_{M}$.

Os programas utilizados nos experimentos para o cálculo de LBP, VAR e LPQ foram obtidos de University of Oulu (OULU, 2011). A implementação do SVM, 
utilizada no último experimento (5) foi obtida de (CHANG; LIN, 2001). Para o cálculo dos atributos derivados da GLCM, foram utilizadas funções disponíveis no MATLAB (MATHWORKS, 2009).

Todos os atributos extraídos da GLCM foram normalizados de modo a terem média nula e desvio padrão unitário. Os histogramas de LBP e LPQ foram também normalizados, dividindo-se cada um de seus valores pela soma total dos valores.

Em face da relativa escassez de segmentos da base de dados, optou-se pela estratégia leave-one-out para a avaliação do desempenho em todos os experimentos.

\subsubsection{Experimento 1 - Avaliação do Impacto da Informação de Contraste}

\section{Objetivo e Procedimento}

O objetivo desse experimento foi avaliar a contribuição relativa dos descritores de textura formados a partir de $L B P_{P, R}$ e $V A R_{P, R}$ em aplicações de classificação de imagens de SR. Especificamente, avaliam-se quatro descritores de textura constituídos:

a) apenas pelo histograma $L B P_{P, R}$,

b) apenas pelo histograma $V A R_{P, R}$,

c) pelo histograma resultante da concatenação dos histogramas individuais de $L B P_{P, R}+V A R_{P, R}, \mathrm{e}$

d) pelo histograma bidimensional que representa a distribuição conjunta textura $L B P_{P, R} / V A R_{P, R}$.

O experimento visa, portanto, determinar a acurácia da classificação associada ao $L B P_{P, R}$ e ao $V A R_{P, R}$ isoladamente (configurações $a$ e $b$ ), bem como a estimar o ganho decorrente de combiná-los num único descritor (configurações $c$ e $d$ ). $\mathrm{O}$ experimento também avalia se ocorre perda importante de acurácia quando se concatenam os histogramas $L B P_{P, R}$ e $V A R_{P, R}$ formando um histograma unidimensional (configuração $c$ ) como alternativa ao histograma bidimensional (configuração $d$ ) que representa a distribuição conjunta de $L B P_{P, R}$ e $V A R_{P, R}$. A configuração $c$ é uma alternativa proposta neste trabalho.

Em todos os casos os valores de $V A R_{P, R}$ foram quantizados em 8 níveis. $\mathrm{O}$ classificador utilizado baseia-se na estatística $G$ (Equação 4) como medida de dissimilaridade.

\section{Resultados e Discussão}

A Tabela 3 apresenta os valores do índice Kappa registrados neste experimento para ambas as aplicações e áreas de estudo considerando apenas versões monoescalares dos descritores de textura. A tabela mostra ainda nas duas colunas à direita a dimensão do descritor em cada caso.

O índice Kappa variou entre 0,69 e 0,81 e entre 0,53 e 0,79 para as áreas de estudo 1 e 2 respectivamente nos experimentos que envolveram o $L B P_{P, R}$ isolado. Nota-se, ainda, que os melhores e piores valores foram alcançados para diferentes 
combinações de $P$ e $R$, nas duas aplicações. Por exemplo, enquanto $(P, R)=(8,1)$ apresentou o pior $(K a p p a=0,69)$ entre os desempenhos medidos para a área de estudo 1, na área de estudo 2 alcançou desempenho muito próximo ao máximo observado (Kappa=0,78). Verifica-se, portanto, que os valores ótimos de $P$ e $R$ dependem da aplicação alvo e o ajuste destes parâmetros pode ter forte impacto sobre o desempenho. Cabe observar que em nossos experimentos os melhores resultados foram alcançados para $P$ igual a 8 ou 16 e para $R$ entre 2 e 3, o que coincide com outros estudos sobre o LBP (OJALA; PIETIKÄINEN; MÄENPÄÄ, 2002).

Tabela 3 - Resultados da classificação para descritores de textura monoescalares

\begin{tabular}{|c|c|c|c|c|c|c|c|c|c|c|c|c|}
\hline \multirow[b]{3}{*}{$P, R$} & \multicolumn{8}{|c|}{ índice Kappa } & \multirow{2}{*}{\multicolumn{4}{|c|}{$\begin{array}{c}\text { \#bins dos } \\
\text { histogramas }\end{array}$}} \\
\hline & \multicolumn{4}{|c|}{ Área de Estudo 1} & \multicolumn{4}{|c|}{ Área de Estudo 2} & & & & \\
\hline & 品 & $\sum^{\frac{N}{2}}$ & $\frac{a}{\frac{1}{2}}$ & 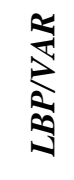 & 今a & 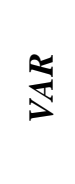 & $\begin{array}{l}\frac{a}{2} \\
\frac{1}{0} \\
\frac{1}{9}\end{array}$ & 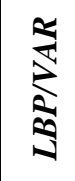 & 今a & $\underset{\Sigma}{\Sigma}$ & $\begin{array}{l}\frac{a}{5} \\
\frac{1}{+} \\
\frac{1}{d}\end{array}$ & 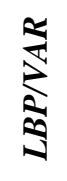 \\
\hline 8,1 & 0,69 & 0,81 & 0,81 & 0,83 & 0,78 & 0,72 & 0,83 & 0,83 & 10 & 8 & 18 & 80 \\
\hline 8,2 & 0,76 & 0,79 & 0,90 & 0,89 & 0,79 & 0,77 & 0,85 & 0,86 & 10 & 8 & 18 & 80 \\
\hline 8,3 & 0,79 & 0,77 & 0,88 & 0,90 & 0,65 & 0,82 & 0,86 & 0,86 & 10 & 8 & 18 & 80 \\
\hline 16,2 & 0,81 & 0,80 & 0,84 & 0,86 & 0,78 & 0,77 & 0,91 & 0,87 & 18 & 8 & 26 & 144 \\
\hline 16,3 & 0,81 & 0,77 & 0,87 & 0,88 & 0,78 & 0,84 & 0,86 & 0,86 & 18 & 8 & 26 & 144 \\
\hline 24,3 & 0,81 & 0,76 & 0,88 & 0,86 & 0,76 & 0,77 & 0,82 & 0,83 & 26 & 8 & 34 & 208 \\
\hline 24,5 & 0,78 & 0,68 & 0,86 & 0,85 & 0,53 & 0,77 & 0,74 & 0,77 & 26 & 8 & 34 & 208 \\
\hline
\end{tabular}

Já para o cálculo de $V_{A} R_{P, R}$ a escolha dos valores de $P$ e $R$ não implicou em diferenças importantes de desempenho. Esta constatação, não surpreende, uma vez que se trata de maneiras alternativas de estimar um mesmo atributo, a variância local. É interessante notar que o desempenho registrado na coluna VAR para ambas as áreas de estudo é, na maioria dos casos, superior ou pouco inferior ao melhor desempenho alcançado a partir de $L B P_{P, R}$ apenas. Além disso, o descritor de textura baseado em $V A R_{P, R}$ é em nossos experimentos mais compacto, isto é, o histograma têm apenas 8 bins, enquanto que o histograma para o $L B P_{P, R}$ possui 10, 18 e 26 bins, respectivamente para $P$ igual a 8,16 e 24 .

Observa-se também na Tabela 3 que as versões combinadas $L B P_{P, R}+V A R_{P, R}$, e $L B P_{P, R} / V A R_{P, R}$ apresentaram resultados consistentemente superiores aos produzidos a partir dos descritores individuais. Em nossos experimentos a combinação trouxe em média um aumento próximo a 0,10 no índice Kappa, para as duas áreas de 
estudo, o que constitui um ganho significativo em aplicações de classificação de imagens de SR. Há novamente neste caso coerência com os resultados registrados em (OJALA; PIETIKÄINEN; MÄENPÄÄ, 2002).

Outra constatação importante é que as duas versões combinadas, $L B P_{P, R}+V A R_{P, R}$, e $L B P_{P, R} / V A R_{P, R}$ apresentaram desempenhos próximos. O descritor proposto neste trabalho, $L B P_{P, R}+V A R_{P, R}$, preservou, portanto, a informação relevante para a classificação contida na versão bidimensional $L B P_{P, R} / V A R_{P, R}$. As colunas mais à direita da tabela mostram que o descritor $L B P_{P, R}+V A R_{P, R}$, têm um número de bins substancialmente menor do que a versão $L B P_{P, R} / V A R_{P, R}$. Essa vantagem implica na simplificação da etapa de classificação, e potencialmente propicia uma maior capacidade de generalização do classificador, ou de outra forma, a redução da demanda por padrões de treinamento para o classificador.

\subsubsection{Experimento 2 - Avaliação de Versões Multiescalares}

\section{Objetivo e Procedimento}

O objetivo desse experimento foi avaliar diversas configurações multiescalares e compará-las com as versões monoescalares que as compõem.

Novamente neste experimento, os valores de $V A R_{P, R}$ foram quantizados em 8 níveis e várias combinações de $P, R$ foram testadas. Como no experimento anterior, o classificador utilizado baseia-se na estatística G (Equação 4) para medida de dissimilaridade.

\section{Resultados e Discussão}

A Tabela 4 apresenta os resultados de experimentos realizados sobre os mesmos conjuntos de dados descritos na seção 3, utilizando as versões multiescalares dos descritores texturais.

Comparando células correspondentes das tabelas 3 e 4, conclui-se que em cerca de $2 / 3$ dos casos a versão multiescalar apresentou desempenho inferior ou igual ao de pelo menos um dos descritores monoescalares que a compõem. O maior ganho no índice Kappa foi de 0,06 observado para o descritor $L B P_{P, R}$ nas configurações $(8,1+8,3)$ e $(8,1+16,2+24,3)$ para a área de estudo 2 . Nas versões de maior desempenho que combinam o $L B P_{P, R}$ e o $V A R_{P, R}$ o ganho máximo foi de 0,03 , medido também na área de estudo 2 para as configurações $(8,2+8,3)$ e $(8,1+8,2+8,3)$. Os resultados mostram que a abordagem multiescalar não trouxe ganhos de acurácia e sugerem, portanto, que a informação de textura relevante para separar as classes nas aplicações de teste pôde ser adequadamente capturada em uma única escala.

Um fator que pode ter afetado os resultados das versões multiescalares é a dimensão dos descritores, particularmente elevada nas versões $L B P_{P, R} / V A R_{P, R}$, como mostram as colunas mais à direita das tabelas 3 e 4 . Pode-se cogitar de que $o$ desempenho associado aos descritores multiescalares poderia eventualmente superar o das versões monoescalares à medida que mais segmentos fossem utilizados para 
caracterizar cada classe. Para testar esta possibilidade seriam, no entanto, necessários mais segmentos do que dispõem os conjuntos de dados utilizados em nossos experimentos, o que é uma dificuldade comum em aplicações reais de interpretação de imagens de SR.

Tabela 4 - Resultados da classificação para descritores de textura multiescalares formados por diferentes combinações de $L B P_{P, R}$ e $V A R_{P, R}$.

\begin{tabular}{|c|c|c|c|c|c|c|c|c|c|c|c|c|}
\hline \multirow[b]{3}{*}{$P, R$} & \multicolumn{8}{|c|}{ índice Kappa } & \multirow{2}{*}{\multicolumn{4}{|c|}{$\begin{array}{c}\text { \#bins dos } \\
\text { histogramas }\end{array}$}} \\
\hline & \multicolumn{4}{|c|}{ Área de Estudo 1} & \multicolumn{4}{|c|}{ Área de Estudo 2} & & & & \\
\hline & $\frac{2}{19}$ & $\sum_{i}^{2}$ & 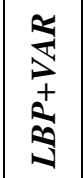 & $\frac{⿱ 亠}{\frac{\pi}{n}}$ & $\frac{0}{19}$ & $\stackrel{\Sigma}{\pi}$ & $\begin{array}{l}\frac{5}{d} \\
\frac{1}{+} \\
\frac{1}{n} \\
\frac{a}{2}\end{array}$ & $\sum_{\substack{n \\
\frac{1}{2}}}^{\frac{2}{2}}$ & $\frac{a}{n}$ & $\sum$ & 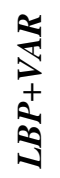 & 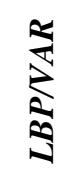 \\
\hline $8,1+8,3$ & 0,81 & 0,82 & 0,88 & 0,88 & 0,84 & 0,79 & 0,86 & 0,86 & 20 & 16 & 36 & 160 \\
\hline $8,2+8,3$ & 0,78 & 0,79 & 0,89 & 0,87 & 0,77 & 0,82 & 0,89 & 0,89 & 20 & 16 & 36 & 160 \\
\hline $8,2+16,3$ & 0,80 & 0,78 & 0,89 & 0,87 & 0,82 & 0,80 & 0,85 & 0,86 & 28 & 16 & 44 & 224 \\
\hline $16,2+24,5$ & 0,80 & 0,78 & 0,88 & 0,86 & 0,79 & 0,78 & 0,82 & 0,80 & 44 & 16 & 60 & 352 \\
\hline $8,2+16,3+24,5$ & 0,79 & 0,80 & 0,88 & 0,86 & 0,79 & 0,77 & 0,82 & 0,82 & 56 & 24 & 78 & 432 \\
\hline $8,1+16,2+24,3$ & 0,84 & 0,82 & 0,88 & 0,87 & 0,84 & 0,76 & 0,91 & 0,86 & 56 & 24 & 78 & 432 \\
\hline $8,1+8,2+8,3$ & 0,81 & 0,81 & 0,89 & 0,86 & 0,84 & 0,79 & 0,89 & 0,89 & 30 & 24 & 54 & 240 \\
\hline
\end{tabular}

Corroborando o que já se observou no experimento anterior, nota-se também neste experimento que as versões $L B P_{P, R}+V A R_{P, R}$ apresentaram desempenho próximo, na verdade geralmente um pouco superior às versões $L B P_{P, R} / V A R_{P, R} \mathrm{em}$ ambas as áreas de estudo.

\subsubsection{Experimento 3 - Comparação de Configurações Monoescalares do $L P Q_{M}$}

\section{Objetivo e Procedimento}

Este experimento teve o propósito de comparar os descritores $L P Q_{M}$ e $L B P_{P, R}$ isoladamente e combinados com a informação de contraste. Como nos experimentos anteriores, o contraste foi representado pelo descritor $V A R_{P, R}$. Os resultados apresentados mais adiante correspondem ao valor de $(P, R)$ do conjunto $\{(8,1),(8,2)$, $(16,2),(16,3)\}$, que produziu o melhor índice Kappa. Para a classificação foi utilizada novamente a estatística $\mathrm{G}$.

Essa análise não considerou a distribuição conjunta de $L P Q_{M}$ e $V A R_{P, R}$. Um histograma bidimensional representando a distribuição conjunta conteria, neste caso, 2550 bins, o que seria excessivo para muitas aplicações práticas. Desta forma, a 
avaliação se restringiu à versão concatenada $L P Q_{M}+V A R_{P, R}$, além do $L P Q_{M}$ isoladamente.

\section{Resultados e Discussão}

Os resultados das classificações das áreas em estudo com o uso do $L P Q_{M}$ são mostrados na Tabela 5, onde também se apresenta a contribuição da informação de contraste.

O índice Kappa variou entre 0,65 e 0,74 na área de estudo 1 e entre 0,74 e 0,86 na área de estudo 2 para o descritor $L P Q_{M}$ utilizado isoladamente. Recorrendo à Tabela 3, verifica-se que para o descritor $L B P_{P, R}$ isolado o índice Kappa variou entre 0,69 e 0,81 para área 1 e entre 0,53 e 0,79 para a área 2.

Tabela 5 - Resultados da classificação das áreas de estudo com o uso dos descritores $L P Q_{M}$ e informações de contraste.

\begin{tabular}{c|c|c|c|c|c|c}
\hline \multirow{2}{*}{$M$} & \multicolumn{4}{|c|}{ índice Kappa } & \multicolumn{2}{|c}{$\begin{array}{c}\text { \#bins dos } \\
\text { histogramas }\end{array}$} \\
\cline { 2 - 5 } & Área de Estudo 1 & Área de Estudo 2 & \multicolumn{2}{|c}{ hom } \\
\cline { 2 - 5 } & LPQ & LPQ+VAR & LPQ & LPQ+VAR & LPQ & LPQ+VAR \\
\hline 3 & 0,74 & 0,87 & 0,82 & 0,91 & & \\
\hline 5 & 0,74 & 0,92 & 0,86 & 0,91 & \multirow{2}{*}{255} & \multirow{2}{*}{263} \\
\hline 7 & 0,65 & 0,89 & 0,74 & 0,86 & & \\
\hline 9 & 0,67 & 0,89 & 0,86 & 0,84 & & \\
\hline
\end{tabular}

Uma análise dos valores relativos à combinação com a informação de variância $\left(V A R_{P, R}\right)$ conduz a conclusão similar. Note-se que o índice Kappa varia numa faixa de valores muito semelhante tanto para $L P Q_{M}+V A R_{P, R}$ como para $L B P_{P, R}+V A R_{P, R}$ em ambas as áreas de estudo.

Em síntese, a comparação com as versões baseadas no $L B P_{P, R}$ mostrou que a maior complexidade do cálculo dos descritores derivados do $L P Q_{M}$ não foi recompensada por benefícios correspondentes em termos de performance.

\subsubsection{Experimento 4 - Comparação de Configurações Multiescalares do $L P Q_{M}$}

\section{Objetivo e Procedimento}

Avaliaram-se nesse experimento configurações multiescalares $L P Q_{M}$ e suas combinações com a informação de contraste. Como nos experimentos anteriores, os valores de $V A R_{P, R}$ foram quantizados em 8 níveis. Pelos motivos já arrolados, a análise considerou apenas a versão concatenada $L P Q_{M}+V A R_{P, R}$, além do $L P Q_{M}$ isoladamente. Os valores de $P$ e $R$ utilizados no cálculo do VAR correpondem aos valores que produziram o melhor resultado no experimento 2 . 


\section{Resultados e Discussão}

Os resultados das classificações das áreas em estudo com o uso do $L P Q_{M}$ são mostrados na Tabela 6 .

Coerentemente com os resultados do experimento 2, não se observou nesse experimento uma superioridade flagrante das versões multiescalares sobre as correspondentes versões monoescalares do $L P Q_{M}$. Como já se aventou anteriormente, isso ocorreu provavelmente por que a textura de cada uma das classes pôde ser adequadamente caracterizada em uma única escala em ambas as áreas de estudo.

Tabela 6 - Resultados da classificação das áreas de estudo com o uso dos descritores $L P Q_{M}$ mutiescalares associados a informações de contraste.

\begin{tabular}{c|c|c|c|c|c|c}
\hline \multirow{2}{*}{$M$} & \multicolumn{4}{|c|}{ índice Kappa } & \multicolumn{2}{c}{$\begin{array}{c}\text { \#bins dos } \\
\text { histogramas }\end{array}$} \\
\cline { 2 - 5 } & Área de Estudo 1 & Área de Estudo 2 & \multicolumn{2}{c}{ LPQ } & LPQ+VAR \\
\cline { 2 - 5 } & $\mathbf{L P Q}$ & $\mathbf{L P Q + V A R}$ & $\mathbf{L P Q}$ & $\boldsymbol{L P Q + V A R}$ & $\boldsymbol{L P Q}$ \\
\hline $3+5$ & 0,79 & 0,90 & 0,84 & 0,89 & 500 & 508 \\
\hline $5+9$ & 0,74 & 0,91 & 0,84 & 0,89 & 500 & 508 \\
\hline $3+9$ & 0,77 & 0,89 & 0,86 & 0,93 & 500 & 508 \\
\hline $7+9$ & 0,65 & 0,87 & 0,81 & 0,84 & 500 & 508 \\
\hline $3+5+7+9$ & 0,80 & 0,89 & 0,86 & 0,91 & 1020 & 1028 \\
\hline
\end{tabular}

3.2.5 Experimento 5 - Comparação entre $\mathrm{LBP}_{\mathrm{P}, \mathrm{R}}, \mathrm{LPQ}_{\mathrm{M}}$ e GLCM

\section{Objetivo e Procedimento}

Nos experimentos anteriores o melhor compromisso entre desempenho e complexidade foi alcançado pelos descritores $L B P_{P, R}+V A R_{P, R}$ e $L P Q_{M}+V A R_{P, R}$. O objetivo do experimento 5 é comparar esses descritores com descritores derivados da GLCM, os mais largamente utilizados pela comunidade de SR para caracterizar texturas.

Para que a comparação seja imparcial convém que se utilize um mesmo classificador para todos os descritores. Um classificador baseado na estatística $G$ não se adéqua a esta comparação, uma vez que os descritores GLCM não são histogramas, mas escalares organizados em vetores de atributos. O classificador escolhido para a realização dos testes foi a SVM, na sua forma one-against-all.

Foram analisadas as combinações monoescalares de LBP e LPQ em conjunto com a informação de contraste, à semelhança dos experimentos anteriores. Foram testadas configurações diferentes para o cálculo da GLCM, especificamente, o número de níveis de cinza $\left(N_{\mathrm{g}} \in\{128,64,32,16\}\right)$ da imagem, a distância que caracteriza o operador de posição $(d \in\{1,2,3\})$ Das matrizes de co-ocorrência calculadas para as orientações $(\theta) 0^{\circ}, 45^{\circ}, 90$ e, $135^{\circ}$ extraíram-se os atributos energia, entropia, homogeneidade, contraste, variância e correlação para caracterizar as texturas. 


\section{Resultados e Discussão}

Nas Tabelas $7 a$ a $7 c$ são mostrados os valores do índice Kappa medidos nos experimentos. A Figura 5 mostra os melhores e os piores resultados medidos para cada descritor.

Tabela 7 - Resultados da classificação por SVM a partir dos descritores (a) $L B P_{P, R}+V A R_{P, R},(b) L P Q_{M}+V A R_{P, R}$ e (c) GLCM.

\begin{tabular}{|c|c|c|}
\hline \multirow{2}{*}{$P, R$} & \multicolumn{2}{|c|}{$\begin{array}{c}\text { índice Kappa para } \\
L B P+V A R \\
\end{array}$} \\
\hline & \begin{tabular}{|c|} 
Área de \\
Estudo 1 \\
\end{tabular} & \begin{tabular}{|l|} 
Área de \\
Estudo 2 \\
\end{tabular} \\
\hline 8,1 & 0,86 & 0,86 \\
\hline 8,2 & 0,89 & 0,93 \\
\hline 8,3 & 0,89 & 0,93 \\
\hline 16,2 & 0,90 & 0,93 \\
\hline 16,3 & 0,87 & 0,89 \\
\hline 24,3 & 0,86 & 0,89 \\
\hline 24,5 & 0,91 & 0,77 \\
\hline
\end{tabular}

\begin{tabular}{|c|c|c|}
\hline \multirow{2}{*}{$M$} & \multicolumn{2}{|c|}{$\begin{array}{c}\text { índice Kappa para } \\
L P Q+V A R\end{array}$} \\
\hline & $\begin{array}{l}\text { Área de } \\
\text { Estudo } 1\end{array}$ & $\begin{array}{l}\text { Área de } \\
\text { Estudo } 2\end{array}$ \\
\hline
\end{tabular}

\begin{tabular}{c|c|c}
\hline \multirow{2}{*}{$N_{\mathrm{g}}, d$} & \multicolumn{2}{|c}{$\begin{array}{c}\text { índice Kappa para } \\
\text { GLCM }\end{array}$} \\
\cline { 2 - 3 } & $\begin{array}{c}\text { Área de } \\
\text { Estudo 1 }\end{array}$ & $\begin{array}{c}\text { Área de } \\
\text { Estudo 2 }\end{array}$ \\
\hline 16,1 & 0,80 & $\mathbf{0 , 6 6}$ \\
\hline 16,2 & 0,78 & 0,68 \\
\hline 16,3 & $\mathbf{0 , 7 8}$ & $\mathbf{0 , 6 6}$ \\
\hline 32,1 & $\mathbf{0 , 8 4}$ & 0,75 \\
\hline 32,2 & 0,82 & 0,73 \\
\hline 32,3 & 0,82 & 0,78 \\
\hline 64,1 & $\mathbf{0 , 8 4}$ & $\mathbf{0 , 8 2}$ \\
\hline 64,2 & 0,80 & 0,77 \\
\hline 64,3 & 0,82 & 0,67 \\
\hline 128,1 & $\mathbf{0 , 8 4}$ & 0,77 \\
\hline 128,2 & 0,81 & 0,75 \\
\hline 128,3 & 0,82 & 0,68 \\
\hline
\end{tabular}

(c)

A Figura 5 revela uma nítida superioridade dos resultados derivados do LBP e do LPQ em relação aos descritores GLCM. A capacidade de discriminação de cada descritor pode ser avaliada comparando-se os valores máximos do índice Kappa registrados em cada caso. Referente à primeira área de estudo, 0,91 e 0,93 foram os valores máximos obtidos com os descritores LBP e LPQ respectivamente, enquanto que o máximo alcançado a partir da GLCM foi 0,84 . Nos experimentos realizados sobre a área de estudo 2, o índice Kappa atingiu valores de 0,93 e 0,89, respectivamente para o LBP e LPQ, ao passo que o valor máximo observado para os descritores GLCM foi 0,82. É significativo que, na área de estudo 1, o melhor resultado medido para o $\operatorname{GLCM}(\mathrm{Kappa}=0,84)$ foi inferior ao pior resultado registrado para os outros dois descritores (Kappa $=0,85)$. 
Figura 5 - Comparação de resultados da classificação com os descritores LBP, LPQ e GLCM das áreas de estudo.

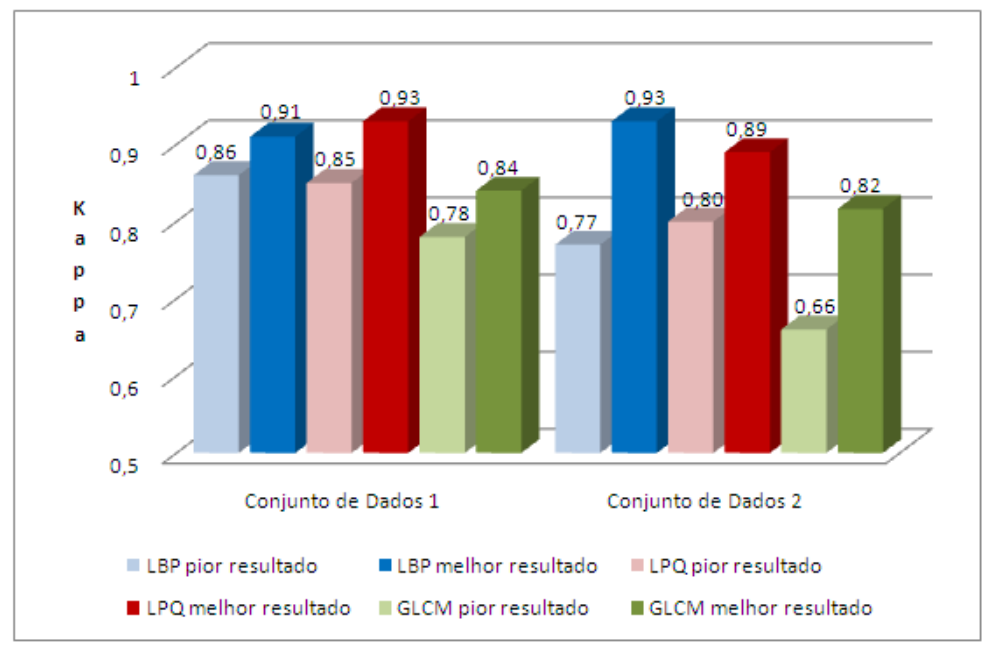

Os valores mínimos observados para cada descritor numa mesma área de estudo são igualmente mais baixos para o GLCM do que para os demais descritores. Ao mesmo tempo em que se registrou 0,78 como valor mínimo para os descritores GLCM, o menor valor observado para o LBP e LPQ foi 0,86 e 0,85 respectivamente. De modo semelhante na área de estudo 2 , o menor valor obtido a partir do GLCM foi 0,66, enquanto o LBP e o LPQ não caíram abaixo de 0,77 e 0,80 respectivamente. Estes resultados sugerem, portanto, que a escolha dos parâmetros derivados da GLCM $\left(N_{\mathrm{g}}, d\right.$ e $\left.\theta\right)$ é mais crítica do que a escolha dos parâmetros do LBP $(P$ e $R)$ ou do LPQ $(M)$.

As diferenças do índice Kappa observadas, especialmente levando-se em conta que ocorreram em torno de valores elevados, permitem concluir que as variantes do LBP e do LPQ foram significativamente superiores aos descritores derivados do GLCM em nossos experimentos.

Encerrando esta seção, cumpre observar que os resultados obtidos pela SVM para os descritores LBP e LPQ não diferem substancialmente dos resultados registrados nos experimentos anteriores em que a estatística $\mathrm{G}$ foi a métrica básica usada na classificação. Os resultados encorajam o uso da SVM para a classificação de imagens de SR a partir de atributos de textura representados por histogramas de padrões binários locais combinados com atributos espectrais ou de forma, o que, como já se observou, provavelmente resultaria em melhores índices de desempenho dos que os registrados neste estudo. 


\section{CONCLUSÃO}

Neste trabalho, foram avaliadas experimentalmente técnicas baseadas em Padrões Binários Locais (Local Binary Patterns - LBP), Quantização Local de Fase (Local Phase Quantization - LPQ) e na variância para descrição de texturas em imagens de sensores remotos de muito alta resolução espacial - Quickbird-2 e IKONOS-2. Foram analisadas diversas variantes dos descritores nas versões monoe multiescalares.

Os experimentos confirmaram trabalhos anteriores em que se concluiu que o poder de discriminação tanto do LBP quando do LPQ melhora significativamente quando combinados com a informação de contraste.

$\mathrm{O}$ presente estudo propôs ainda um descritor de textura formado pela concatenação do histograma dos códigos binários locais com o histograma de variância local. A análise experimental indicou que o descritor proposto, embora muito mais compacto, preservou o poder de discriminação dos histogramas bidimensionais introduzidos em trabalhos anteriores e que representam a distribuição conjunta dos códigos binários e da variância local.

Cabe mencionar que não se observou superioridade significativa das versões multiescalares dos descritores LBP e LPQ sobre as correspondentes versões monoescalares.

Diferentemente do que a literatura registra para aplicações de reconhecimento facial, os índices de desempenho medidos em nossos experimentos para os descritores derivados do LPQ e do LBP foram muito próximos. Em outras palavras, a maior complexidade do cálculo do LPQ não se traduziu em ganhos correspondentes de desempenho.

Por fim, o trabalho comparou os descritores propostos com os descritores derivados da GLCM introduzidos por Haralick que são largamente utilizados para descrição de texturas em aplicações de sensoriamento remoto. Os experimentos mostraram uma significativa superioridade dos descritores baseados em códigos binários locais sobre os descritores de Haralick.

\section{REFERÊNCIAS BIBLIOGRÁFICAS}

AHONEN, T.; HADID, A.; PIETIKÄINEN, M. Face Description with Local Binary Patterns: Application to Face Recognition. IEEE Trans. Pattern Analysis and Machine Intelligence, vol. 28(12), p. 2037-2041, 2006.

AHONEN, T. et al. Recognition of Blurred Faces Using Local Phase Quantization. Proc. 19th International Conference on Pattern Recognition (ICPR 2008), Tampa, 2008, p. 1-4.

BAZI, Y.; MELGANI, F. Toward an Optimal SVM Classification System for Hyperspectral Images. Geoscience and Remote Sensing, IEEE Transactions on vol 44(2), DOI: 10.1109/TGRS.2006.880628, 2006, p.3374-3385.

BOSCHI, L.S.;GALO, M.L.B.T. Análise de incerteza na representação de classes de cobertura do solo urbano resultantes da aplicação de uma rede neural artificial, Boletim de Ciências Geodésicas, vol. 13(1), p. 22-41, 2007. 
BRODATZ, P. Textures: A Photographic Album for Artists and Designers. Dover, 1966.

CHAN, C. H. et al. (Multiscale) local phase quantization histogram discriminant analysis with score normalisation for robust face recognition. Proc. 1st IEEE Workshop on Video-Oriented Object and Event Classification, 2009, Kyoto, Japan, p. 633-640.

CHANG, C.C.; LIN, C. J. (2001). LIBSVM: a library for support vector machines. Disponível em: $<\mathrm{http}: / / w w w . c s i e . n t u . e d u . t w / \sim c j l i n / l i b s v m>$. Acesso em: $30 \mathrm{de}$ abr. 2011.

CORTES, C.; VAPNIK, V.N. Support-Vector Networks, Machine Learning 20(3), 1995, P. 273-297.

GUO, D.; ATLURI, V.; ADAM, N. Texture-based remote-sensing image segmentation. Proc. International Conference on Multimedia and Expo, 2005, Amsterdam, p. 1472-1475.

HARALICK, R. M.; DINSTEIN, I.; SHANMUGAM, K. Textural features for image classification. IEEE Transactions on Systems, Man, and Cybernetics, vol. 3, p. 610-621, 1973.

HAY, G. J.; CASTELLAN, G. Geographic Object-Based Image Analysis (GEOBIA): A new name for a new discipline. Geographic Object-Based Image Analysis - Lecture Notes in Geoinformation and Cartography, Section 1, 75-89, 2008.

HEIKKILÄ, J.; OJANSIVU, V. Methods for Local Phase Quantization in BlurInsensitive Image Analysis. Proc. International Workshop on Local and NonLocal Approximation in Image Processing (LNLA 2009), Tuusula, Finland, p. 104-111.

HEIKKILÄ, J.; OJANSIVU, V.; RAHTU, E. Improved blur insensitivity for decorrelated local phase quantization. Proc. 20th International Conference on Pattern Recognition (ICPR 2010), Istanbul, Turkey, p. 818-821.

HU, X.; TAO, C.V.; PRENZEL, B. 2005. Automatic segmentation of highresolution satellite imagery by integrating texture, intensity, and color features. Photogrammetric Engineering \& Remote Sensing, vol. 71(12), p. 1399-1406.

HUANG, C.; DAVIS, L. S.; TOWNSHED, J. R. G. An assessment of support vector machines for land cover classification. International Journal of Remote Sensing, 2002, 23, p. 725-749.

JYOTHI, B. N.; BABU, G.R.; KRISHNA, I.V.M. Object Oriented and Multi-Scale Image Analysis: Strengths, Weaknesses, Opportunities and Threats-A Review. Journal of Computer Science 4 (9), p. 706-712, 2008.

KIM, M.; MADDEN, M.; WARNER, T.A. Forest type mapping using objectspecific texture measures from multispectral Ikonos imagery: Segmentation quality and image classification issues, Photogrammetric Engineering and Remote Sensing, vol. 75(7), 2009, p. 819-829. 
LU, D.; WENG, Q. Survey of image classification methods and techniques for improving classification performance. International Journal of Remote Sensing, 28(5), p. 823-870, 2007.

LUCIEER, A.; STEIN, A.; FISHER, P. Multivariate texture-based segmentation of remotely sensed imagery for extraction of objects and their uncertainty, International Journal of Remote Sensing 26(14), 2005, p. 2917-2936.

LUCIEER, A.; STEIN, A. Texture-based landform segmentation of LiDAR imagery. International Journal of Applied Earth Observation and Geoinformation, vol. 6(3-4), p. 261-270, 2005.

MATHWORKS, MATLAB Image Processing Toolbox, version 6.3, 2009.

MUSCI, M. et al. Padrões Binários Locais na Classificação de Imagens de Sensoriamento Remoto In: XV Simpósio Brasileiro de Sensoriamento Remoto, 2011, Curitiba. Anais... São José dos Campos: INPE, p.7651-7658, 2011.

OJALA, T.; PIETIKÄINEN, M.; HARWOOD, D. A Comparative study of Texture Measures with Classification Based on Feature Distributions, Pattern Recognition 29 (1), p. 51-59, 1996.

OJALA, T.; PIETIKÄINEN, M.; MÄENPÄÄ, T. Multiresolution gray scale and rotation invariant texture classification with local binary patterns. IEEE Transactions on Pattern Analysis and Machine Intelligence, vol. 24(7), p. 971987, 2002.

OJANSIVU, V.; HEIKKILÄ, J. Blur insensitive texture classification using local phase quantization. Proc. 3rd International Conference on Image and Signal Processing (ICISP 2008), Cherbourg-Octeville, France, p. 236-243.

OJANSIVU, V.; RAHTU, E.; HEIKKILÄ, J. Rotation invariant blur insensitive texture analysis using local phase quantization. Proc. 19th International Conference on Pattern Recognition (ICPR 2008), Tampa, FL, 2008, p. 4.

ORKHONSELENGE, T. Texture based segmentation of remotely sensed imagery for identification of geological units. M.Sc. thesis, International Institute for Geo-information Science and Earth Observation, Enschede, 2004.

OULU. University of Oulu - Department of Computer Science and Engineering, LBP Disponível em: <http://www.cse.oulu.fi/MVG/Downloads/LBPMatlab >.

LPQ Disponível em: < http://www.cse.oulu.fi/Downloads/LPQMatlab >. Acesso em: 16 mar. 2011.

PAL, M.; FOODY, G.M. Feature Selection for Classification of Hyperspectral Data by SVM. Geoscience and Remote Sensing, IEEE Transactions on vol. 48(5), DOI: 10.1109/TGRS.2009.2039484, 2010, p. 2297-2307.

PEÑA-BARRAGÁN, J.M. et al. Object-based crop identification using multiple vegetation indices, textural features and crop phenology. Remote Sensing of Environment, vol. 115(6), p. 1301-1316, 2011.

PETROU, M.; SEVILlA, P.G. Dealing with Texture, Wiley, 2006, ISBN: 97804700262812006.

PMPS - Prefeitura Municipal da Cidade de Sao Paulo, InfoLocal. Disponível em: $<$ http://infolocal.prefeitura.sp.gov.br/>. Acesso em 19 abr. 2009. 
RUBIO, T.J. et al. A hierarchical context-based textured image segmentation algorithm for aerial images. Proc. 2nd International Workshop on Texture Analysis and Synthesis, Copenhagen, Denmark, 2001, p. 117-121.

SOKAL, R. R.; ROHLF, F.J. Introduction to Biostatistics. 2nd. W.H. Freeman and Co, New York, 1987.

SONG, C.; YANG, F.; LI, P. Rotation Invariant Texture Measured By Local Binary Pattern For Remote Sensing Image Classification. Proc. Second International Workshop On Education Technology And Computer Science (Etcs 2010), Wuhan, China, P. 3-6, ISBN: 978-1-4244-6388-6.

WANG, A.; WANG, S.; LUCIEER, A. Segmentation of multispectral highresolution satellite imagery based on integrated feature distributions. International Journal of Remote Sensing 31(6), 2010, p. 1471-1483.

WANG, R.; HU, Y.; ZHANG, X. Extraction of Road Networks Using PanSharpenede Multispectral and Panchromatic QuickBird Images. 2005, Geomatica 59(3), p. 263-273.

WANG, S.; WANG, A. Segmentation of high-resolution satellite imagery based on feature combination. The International Archives of the Photogrammetry, Remote Sensing and Spatial Information Sciences. Vol. XXXVII. Part B4. Beijing 2008.

ZHANG, R.; ZHU, D. Study of land cover classification based on knowledge rules using high-resolution remote sensing images. 2011, Expert Systems with Applications 38(4), p. 3647-3652.

(Recebido em agosto de 2011. Aceito em novembro de 2011.) 